EPJ Web of Conferences 49, 18027 (2013)

DOI: $10.1051 /$ epjconf/20134918027

(C) Owned by the authors, published by EDP Sciences, 2013

\title{
Search for the Standard Model Higgs boson produced in association with a vector boson and decaying to bottom quarks with the ATLAS detector
}

\author{
Heather M. Gray ${ }^{1, a}$, Tim Scanlon ${ }^{1, b}$, and Gabriel Facini ${ }^{1, c}$ on behalf of the ATLAS collaboration. \\ ${ }^{1}$ CERN
}

\begin{abstract}
This note presents an updated search with the ATLAS experiment for the Standard Model Higgs boson produced in association with a $W$ or $Z$ boson and decaying to $b \bar{b}$, using $4.7 \mathrm{fb}^{-1}$ of LHC data at $\sqrt{s}=$ $7 \mathrm{TeV}$ and $13.0 \mathrm{fb}^{-1}$ at $\sqrt{s}=8 \mathrm{TeV}$. The search is performed using events containing zero, one or two electrons or muons targeting the three decay modes $Z H \rightarrow v \bar{v} b \bar{b}, W H \rightarrow \ell v b \bar{b}$ and $Z H \rightarrow \ell^{+} \ell^{-} b \bar{b}$. No significant excess is observed. For $m_{H}=125 \mathrm{GeV}$, the observed (expected) upper limit on the cross section times the branching ratio is found to be 1.8 (1.9) times the Standard Model prediction. The production of diboson pairs, $W Z$ and $Z Z$, with a $Z$ boson decaying to $b \bar{b}$, has been observed with a significance of 4.0 standard deviations at a rate compatible with the Standard Model expectation.
\end{abstract}

\section{Introduction}

The search for the Standard Model (SM) Higgs boson is a central component of the physics program at the Large Hadron Collider (LHC). Recently, the observation of a boson decaying to a pair of photons or massive vector bosons has been reported by the ATLAS [1] and CMS [2] collaborations. Determining the nature of this boson - whether it is indeed the SM Higgs boson - is now one of the most important questions in particle physics.

A SM Higgs boson with a mass of about $125 \mathrm{GeV}$ would be accessible in both bosonic and fermionic decay channels at the LHC. Observing the decay into fermions is vital to determine if the new boson is compatible with a SM Higgs boson. In particular, the decay to $b$-quarks plays an important role since this is expected to be the dominant decay mode at this mass $(B R(H \rightarrow b \bar{b}) \approx 58 \%$ [3] $)$. Therefore an observation in this channel is crucial in order to provide a direct constraint on the dominant decay mode.

The Tevatron experiments have reported an excess of approximately three standard deviations in the search for the Higgs boson in the $b \bar{b}$ decay mode [4]. The CMS collaboration set expected and observed upper limits on the production cross section times branching ratio of 1.6 and 2.1 times the SM expectation [2].

This note presents an update of the results reported in Ref. [5] on the search for the Higgs boson decaying to a pair of $b$-quarks with the Higgs boson produced in association with a $W$ or $Z$ boson in the decay channels $Z H \rightarrow v \bar{v} b \bar{b}, W H \rightarrow \ell v b \bar{b}$ and $Z H \rightarrow \ell^{+} \ell^{-} b \bar{b}$, where $\ell$ refers to either an electron or a muon. It uses $4.7 \mathrm{fb}^{-1}$ of data at $\sqrt{s}=7 \mathrm{TeV}$ and $13.0 \mathrm{fb}^{-1}$ of data at $\sqrt{s}=8 \mathrm{TeV}$,

\footnotetext{
ae-mail: heather.gray@cern.ch

be-mail: tim.scanlon@cern.ch

ce-mail: gabriel.facini@cern.ch
}

delivered by the LHC in 2011 and 2012 respectively. Full details can be found in Ref. [6].

\section{Selection}

Three categories of leptons are used and are denoted in increasing order of purity as loose, medium and tight leptons. Events containing no loose leptons are assigned to the 0 -lepton channel. Those with one tight and no additional loose leptons are studied in the 1-lepton channel. Events with one medium and one additional loose lepton of the same flavour but opposite charge are assigned to the 2-lepton channel.

Jets are reconstructed using the anti- $k_{\mathrm{t}}$ algorithm [7] with a radius parameter $R=0.4$. Jets are required to have $p_{T}>20 \mathrm{GeV}$ and $|\eta|<4.5$, the two jets used to reconstruct the Higgs boson candidate are required to satisfy $|\eta|<2.5$ and the leading jet is required to have $p_{T}>45 \mathrm{GeV}$.

Jets originating from $b$-quarks are identified using the MV1 $b$-tagging algorithm [8-10], tuned to provide an approximate efficiency of $70 \%$ for $b$-jets and rejection factors of $150(5)$ for light $(c)$ jets. Events are required to contain exactly two $b$-tagged jets, which are used to reconstruct the mass of the Higgs boson candidate, $m_{b \bar{b}}$. In 3-jet events, the leading two jets are required to be $b$-tagged.

The missing transverse energy, $E_{\mathrm{T}}^{\text {miss }}$, is measured as the negative vector sum of the transverse momenta associated with cluster energies in the calorimeters with $|\eta|<4$.9. A track-based missing transverse momentum, $p_{\mathrm{T}}^{\text {miss }}$, is calculated as the negative vector sum of the transverse momenta of tracks associated to the primary vertex. The transverse momentum of the vector boson, $p_{\mathrm{T}}^{V}$, where $V$ jointly denotes the $\mathrm{W}$ and and the $\mathrm{Z}$ boson, is reconstructed as the $E_{\mathrm{T}}^{\text {miss }}$ in the 0-lepton channel, the vector 
sum of the lepton and the $E_{\mathrm{T}}^{\mathrm{miss}}$ in the 1-lepton channel $\left(p_{\mathrm{T}}^{W}\right)$ and the vector sum of the two leptons in the 2-lepton channel $\left(p_{\mathrm{T}}^{Z}\right)$. Events containing zero leptons are split into six categories depending on whether they contain two or three jets and on the amount of missing transverse energy. Events containing one or two leptons are divided into five categories of $p_{\mathrm{T}}^{V}$. Topological cuts are used to enhance the signal and reject backgrounds.

The multijet background in the 0-lepton channel is suppressed by cuts on $E_{\mathrm{T}}^{\mathrm{miss}}, p_{\mathrm{T}}^{\mathrm{miss}}$ and their directions with respect to each other and the reconstructed jets. Requirements are made on $E_{\mathrm{T}}^{\mathrm{miss}}$ and the transverse mass of the $W$ boson, $m_{\mathrm{T}}^{W}$, in the 1-lepton channel to select events consistent with the presence of a $W$ boson. The top background is reduced in the 2-lepton channel by cuts on the di-lepton invariant mass $\left(m_{\ell \ell}\right)$ and $E_{\mathrm{T}}^{\mathrm{miss}}$

\section{Signal and Background Modeling}

The dominant backgrounds are top, $W+$ jet and $Z+$ jet production with $Z+$ jet production. The latter is the largest for the 2-lepton channel, the others for the 1-lepton channel and all three make an approximately equal contribution to the 0-lepton channel. Most background shapes are taken from simulation and normalised using control regions in data except for the multijet background, which is estimated entirely from data, and the diboson background for which both normalisation and shape are taken from simulation.

Monte Carlo (MC) event samples produced with the full ATLAS detector simulation [11] based on the GEANT4 [12] program, corrected for all known detector effects, are used to model the Higgs boson signal and most backgrounds. The total production cross sections and associated uncertainties, computed at next-to-leading order (NLO), are taken from Ref. [13] with NLO corrections as a function of the transverse momentum of the vector boson, $p_{\mathrm{T}}^{V}[14,15]$. The decay branching ratios are calculated with Hdecay [3].

Control regions are used to normalise the top background from data in the 1- and 2-lepton channels. In the 1-lepton channel the control region is defined by requiring an additional jet, and for the 2-lepton channel by inverting the requirements on $m_{\ell \ell}$ and $E_{\mathrm{T}}^{\mathrm{miss}}$. Control distributions for the $V+$ jets background are categorised by the number of $b$-tagged jets. The 0 -tag and 1-tag control regions contain exactly 0 or $1 b$-tagged jets.

The normalisations of the $V+c$-jet and $V+$ light-jet backgrounds are determined by a maximum likelihood fit to control and signal regions in the 1- and 2-lepton channels. Events containing 0,1 and $2 b$-tagged jet and the top control regions of each channel are used. This flavour fit exploits the fact that the $b$-tagging has very different efficiencies for $b, c$ and light jets, such that the flavour composition varies significantly with the number of $b$-tagged jets. The normalisations of $Z+$ light, $Z+c, Z+b, W+$ light, $W+c$, $W+b$ and top are allowed to float in the fit. The distributions of the signal regions for each of the three channels after having been normalised by the result of the flavour fit are shown in Fig. 1 for the $\sqrt{s}=8 \mathrm{TeV}$ data.

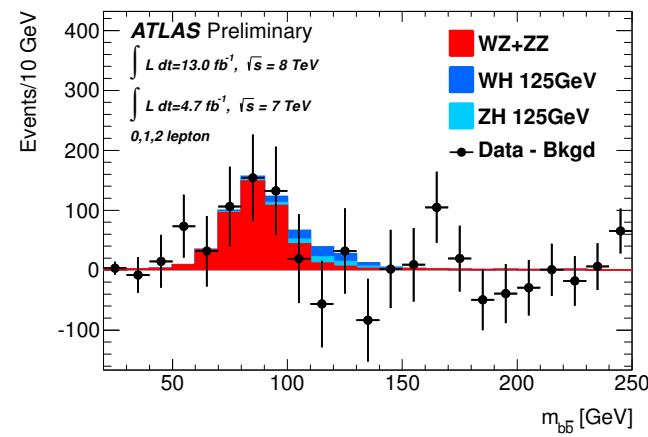

Figure 2. The $m_{b \bar{b}}$ distribution after subtraction of all backgrounds except diboson processes and SM $W H$ and $\mathrm{ZH}$ production. The MC backgrounds are normalised according to the results of the global fit. Only statistical uncertainties are shown. From [6].

Systematic uncertainties are determined on all experimental and theoretical sources. The most significant are on the b-tagging efficiency $(\sim 7 \%)$, the calibration of the jet energy $(\sim 7-20 \%)$ and the statistical uncertainty on the $\mathrm{MC}(\sim 4-8 \%)$.

\section{Results}

The statistical analysis of the data employs a binned profile likelihood function. The categories that enter the profile likelihood fit are the 16 individual $2 b$-tag signal regions and the top control regions for the 1- and 2-lepton channels. A signal strength parameter, $\mu$, scales the expected Standard Model Higgs boson production cross section. The dependence of the signal and background predictions on the systematic uncertainties is described by nuisance parameters. The normalisation of the top, $Z+b$-jet and $W+b$-jet backgrounds float without constraint in the fit, while the other backgrounds have their uncertainty applied as a constraint.

Diboson production with a $Z$ boson decaying to a pair of $b$-quarks and produced with either a $W$ or $Z$ boson has a very similar signature but with lower $p_{\mathrm{T}}^{Z}$ and $m_{b \bar{b}}$ and a cross section $\sim 5$ times larger than Higgs associated production. Therefore a separate fit was made to validate the analysis procedure. In the fit the normalisation of the diboson contribution is allowed to vary with a multiplicative scale $\mu_{D}$ with respect to the SM expectation.

Figure 2 shows the distribution in data after subtracting all backgrounds except the diboson and SM Higgs contribution. An excess in the data compared to the background is observed at the expected mass for the diboson signal. We measure $\mu_{D}=1.05 \pm 0.32$ corresponding to a significance of $4.0 \sigma$ and agrees with the SM expectation of $\mu_{D}=1$.

The profile likelihood fit with the Higgs boson signal strength floating is performed with the diboson contribution fixed to its SM expectation. Figure 3 shows the results for the 95\% CL exclusion limits on the Higgs boson production cross section in the mass range $110-130 \mathrm{GeV}$. The 


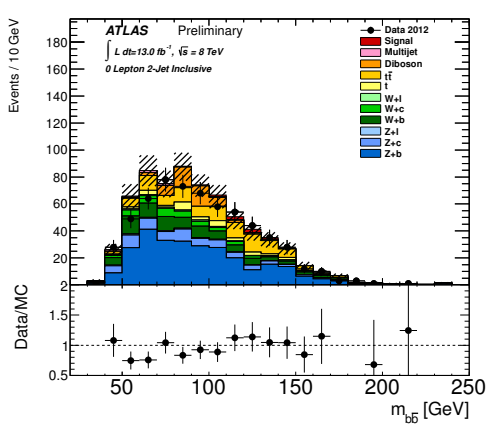

(a) 0-lepton, 2-tag, 2-jets

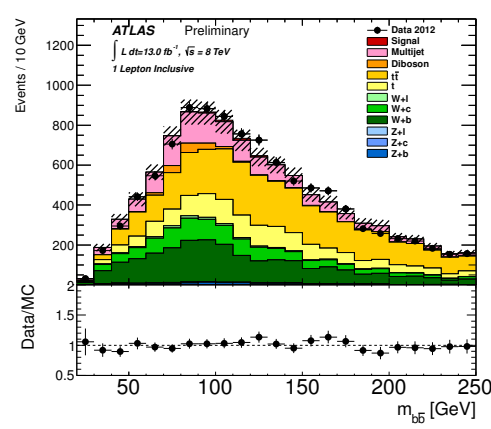

(b) 1-lepton, 2-tag

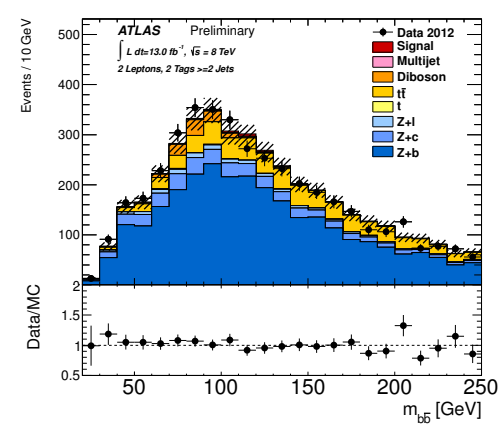

(c) 2-lepton, 2-tag

Figure 1. The $m_{b \bar{b}}$ signal distributions integrated over the bins of $p_{\mathrm{T}}^{V}$. The error bands indicate the size of the combined statistical and systematic uncertainty before the profile likelihood fit. From [6].

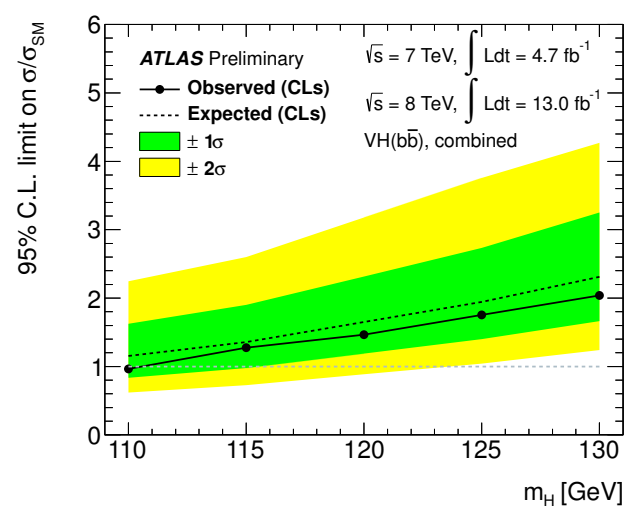

Figure 3. Expected (dashed) and observed (solid) CL limit on the normalised signal strength as a function of $m_{\mathrm{H}}$ for the combination of the $\sqrt{s}=7 \mathrm{TeV}$ and $\sqrt{s}=8 \mathrm{TeV}$ datasets. From [6].

observed limit for the combination of the $\sqrt{s}=7 \mathrm{TeV}$ and $\sqrt{s}=8 \mathrm{TeV}$ datasets is 1.8 times the Standard Model expectation with an expected limit of 1.9 times the Standard Model. The combination of both datasets yields an observed (expected) $p_{0}$ value of $0.64(0.15)$. We measure $\mu=$ $-2.7 \pm 1.1$ (stat.) \pm 1.1 (syst.), $\mu=1.0 \pm 0.9$ (stat.) \pm 1.1 (syst.) and $\mu=-0.4 \pm 0.7$ (stat.) \pm 0.8 (syst.) in the $\sqrt{s}=7 \mathrm{TeV}$, $\sqrt{s}=8 \mathrm{TeV}$ and combined datasets.

\section{Summary}

A search for the Standard Model Higgs boson produced in association with a $W$ or $Z$ boson, using the decay channels $Z H \rightarrow v \bar{v} b \bar{b}, W H \rightarrow \ell v b \bar{b}$ and $Z H \rightarrow \ell^{+} \ell^{-} b \bar{b}$ has been presented using $4.7 \mathrm{fb}^{-1}$ of $p p$ collision data recorded at $\sqrt{s}=7 \mathrm{TeV}$ and $13.0 \mathrm{fb}^{-1}$ of data recorded at $\sqrt{s}=8 \mathrm{TeV}$. No significant excess is observed. For $m_{H}=125 \mathrm{GeV}$, the observed (expected) upper limit on the cross section times the branching ratio is found to be 1.8 (1.9) times the Standard Model prediction for the combination of the $\sqrt{s}=7 \mathrm{TeV}$ and $\sqrt{s}=8 \mathrm{TeV}$ datasets.

\section{References}

[1] Phys.Lett. B716, 1 (2012), 1207.7214

[2] Phys.Lett. B716, 30 (2012), 1207. 7235

[3] A. Djouadi, J. Kalinowski, M. Spira, Comput. Phys. Commun. 108, 56 (1998)

[4] T. Aaltonen et al. (CDF Collaboration, D0 Collaboration), Phys.Rev.Lett. 109, 071804 (2012), 1207.6436

[5] The ATLAS Collaboration (2012), arXiv: 1207.0210

[6] Tech. Rep. ATLAS-CONF-2012-161, CERN, Geneva (2012)

[7] M. Cacciari, G.P. Salam, G. Soyez, JHEP 04, 063 (2008), 0802. 1189

[8] The ATLAS Collaboration, ATLAS-CONF-2012039 (2012)

[9] The ATLAS Collaboration, ATLAS-CONF-2012040 (2012)

[10] The ATLAS Collaboration, ATLAS-CONF-2012043 (2012)

[11] The ATLAS Collaboration, Eur. Phys. J C70, 823 (2010), 1005.4568

[12] S. Agostinelli et al. (GEANT4), Nucl.Instrum.Meth. A506, 250 (2003)

[13] LHC Higgs Cross Section Working Group, CERN2011-002 (CERN, Geneva, 2011), 1101.0593

[14] LHC Higgs Cross Section Working Group, CERN2012-002 (CERN, Geneva, 2012), 1201 . 3084

[15] G. Ferrera, M. Grazzini, F. Tramontano, Phys.Rev.Lett. 107, 152003 (2011), 1107.1164 